\title{
Units of episodic memory in perceptual recognition
}

\author{
JEFFERY J. FRANKS, CHRISTOPHER J. PLYBON, and PAMELA M. AUBLE \\ Vanderbilt University, Nashville, Tennessee 37240
}

\begin{abstract}
Two studies examined the cognitive units of sentence memory using a perceptual recognition task. Four candidate cognitive units were considered: concepts, propositions, integrated propositions, and nonintegrated propositions. Subjects first received a list of acquisition sentences and then were asked to reproduce sentences presented under a white-noise mask. These masked sentences were replicas of the acquisition sentences, were formed of recombined clauses from the acquisition sentences, or were formed of recombined words from the acquisition sentences. Reproduction accuracy was employed as the dependent measure. Results supported propositions (operationalized by clauses) as cognitive units of episodic memory. No conclusive evidence was obtained for concepts, integrated propositions, or nonintegrated propositions as cognitive units. The utility of perceptual recognition tasks for studying the cognitive units of episodic memory is discussed.
\end{abstract}

Recently, Hannigan, Shelton, Franks, \& Bransford (1980) have examined the role of episodic and semantic memory factors (cf. Tulving, 1972) in facilitating the perceptual recognition of sentences masked by white noise. In this work, experimental groups were presented a list of seemingly unrelated sentences as acquisition materials. These sentences could be made to seem interrelated through knowledge of a framework that gave each of them a more specific contextual meaning. One group of subjects was given the framework during acquisition (framework group). A second group was only presented with the sentences (no-framework group). It was hypothesized that presentation of the sentences would result in encoded episodic representations of the information in the sentences. Knowledge of the framework, however, was expected to add higher order semantic knowledge structures within which episodic information would be organized.

Following acquisition, the effects of these episodic and semantic factors on perceptual recognition were assessed (cf. Jacoby \& Dallas, in press). The perceptual recognition task consisted of presenting sentences masked by white noise and asking subjects to reproduce the sentences. Two types of test sentences were old sentences (which were actually presented during acquisition) and novel-appropriate sentences (which were

This work was supported in part by a National Science Foundation predoctoral fellowship (SMI7720981) to Pamela M. Auble. The authors wish to express their thanks to Tommie Slayden and Kate Bergmann for their help in designing and conducting the experiments. We would also like to thank Chuck Clifton, James Neely, and an anonymous reviewer for their helpful comments and criticism of an earlier draft of this paper. Requests for reprints should be sent to Jeffery J. Franks, Department of Psychology, Vanderbilt University, Nashville, Tennessee 37240. not previously presented but did fit the framework provided during acquisition). It was found that both the framework and no-framework groups were more accurate in reproducing old sentences than a control group that had been given neither the acquisition sentences nor the framework. This finding can be attributed to episodic memory for the sentences by the two acquisition groups. In addition, the framework group demonstrated significantly greater reproduction accuracy than the no-framework group and the control group for novel-appropriate sentences, indicating that semantic relationships can also facilitate the processing of information masked by white noise. Thus, the results obtained by Hannigan et al. (1980) indicate the effectiveness of both episodic and semantic memory factors in a perceptual recognition task.

While the Hannigan et al. (1980) studies demonstrated that episodic memory aided sentence reproduction, the nature of the cognitive representations responsible for the enhanced performance remained unclear. The present work examines in more detail the nature of the cognitive units that are the basis for these episodic facilitation effects. Anderson (Note 1) proposes possible candidates for cognitive units. He discusses three levels: the concept, the proposition, and the schema. Mainly on the basis of recognition and recall data, Anderson concluded that episodic effects are quite likely the result of encoding at a combination of propositional and schematic levels. A number of other researchers have interpreted memory data as providing evidence for propositions as cognitive units (e.g., Anderson, 1974; Graesser, 1978).

Ratcliff and McKoon (1978) used a priming paradigm to examine the types of cognitive units operative in a short-term priming task. They proposed three possible structures for the unit of priming: (1) a single unit 
for each sentence with all parts equally closely interrelated, (2) connected propositions, and (3) verbatim representations of the sentences. The nature of the cognitive unit was assessed by recognition reaction time for words from acquisition sentences. Results demonstrated that a noun from a given proposition of an acquisition sentence primed another noun from the same proposition to a significantly greater extent than a noun from another proposition in the same sentence. This was cited as evidence for the proposition as a cognitive unit. These results corroborate the previously mentioned memory data that found evidence supporting propositions as cognitive units of memory.

The present experiments are designed to provide a more detailed examination of the cognitive units underlying episodically enhanced perceptual recognition. The inquiry focuses on four possible levels of cognitive units that might be involved in episodic memory: concept, proposition, comprehended sentence, and noncomprehended sentence representations. These possible units are operationalized in this study by sentences formed from two independent clauses connected by "because" that vary in their comprehensibility. An example of a relatively comprehensible sentence would be "The haystack was important because the parachute cloth ripped," and a relatively incomprehensible sentence would be "The bagpipe split because the clock stopped." For present purposes, the content words in the sentences are considered to correspond to concept units, and clauses correspond to propositions. ${ }^{1}$ Comprehended sentences are considered to be represented by structures of integrated propositions corresponding to whole comprehensible sentences. This type of representation might be considered to be a limited case of "schema" units (e.g., as considered by Anderson, Note 1) in that it makes the distinction between propositions per se and higher order integrated structures of multiple propositions. Finally, noncomprehended sentences are considered to be represented by the structures of two propositions that are not integrated (or, more simply, nonintegrated propositions). That is, these units refer to the possibility of single episodic representations of two conjoined clauses of incomprehensible sentences for which no integrated representation of the separate propositions is formed.

\section{EXPERIMENT 1}

In overview, the experiment consists of an acquisition phase followed by a perceptual recognition test involving reproduction of sentences under a white-noise mask. Both acquisition and test stimuli consist of comprehensible and incomprehensible two-clause sentences. The logic of the design is to hold certain levels of possible cognitive units invariant across acquisition and test while other possible units are varied. In particular, in Experiment 1, both the concept and propositional units are always held invariant between acquisition and test, whereas comprehended and noncomprehended sentences (integrated propositions and nonintegrated propositions) are either held invariant or are altered between acquisition and test. The concepts and propositions are held invariant by using test sentences com. posed of clauses (and therefore, words) that actually occurred in acquisition sentences. Integrated propositions either are held invariant by presenting test sentences that are copies of comprehensible acquisition sentences or are altered by presenting test sentences that are recombinations of clauses from acquisition sentences. Likewise, units composed of nonintegrated propositions either are held invariant by presenting test sentences that are copies of incomprehensible acquisition sentences or are varied by recombining clauses from the acquisition sentences to form new test sentences.

To assess episodic memory effects, all test conditions receiving acquisition will be compared with a baseline condition. Subjects in the baseline condition will receive the white-noise test, but they will not receive prior acquisition. In general, the hypothesis is that if a given level of cognitive unit is an effective mediator of perceptual recognition, then reproduction accuracy will increase when that unit is held invariant between acquisition and test. More specifically, three expectations follow from the general hypothesis: (1) If concepts and/or propositions are effective episodic units, then all conditions receiving acquisition will show facilitated reproduction of test sentences when compared with baseline because words and clauses are invariant across acquisition and test. (2) If integrated or nonintegrated propositions are units of episodic memory, then test sentences that are replicas of acquisition sentences will be reproduced more accurately than sentences composed of recombinations of the clauses from these acquisition sentences.

\section{Method}

Subjects. Thirty-six undergraduate students at Vanderbilt University participated in this experiment as part of an introductory psychology course requirement.

Materials. The set of acquisition sentences was composed of the 20 difficult-to-comprehend sentences used by Auble, Franks, and Soraci (1979) plus 6 additional difficult-to-comprehend sentences generated by the authors. An example of a difficultto-comprehend sentence would be "The breakfast was excellent because the thread was sticky." Each of these 26 sentences had been created so that an additional word or short phrase existed that provided an appropriate context within which the sentence could be understood. For the above sentence, "spiderweb" would be the word enabling comprehension of the sentence (see Auble et al., 1979, for a list of these sentence-word pairs). In the present study, the difficult-to-comprehend sentences were made comprehensible by inserting the cue at a meaningful point in the sentence. For example, a sentence such as "The haystack was important because the cloth ripped," with the word "parachute," would become "The haystack was important because the parachute cloth ripped." A complete list of these altered sentences is given in the appendix. This set of 26 comprehensible 
sentences was used as the acquisition materials in the comprehensible acquisition condition.

The sentences used in the incomprehensible acquisition condition were formed from the same clauses as the comprehensible acquisition sentences. However, these clauses were recombined across sentences in such a way that incomprehensible sentences of the form "Clause 1 because Clause 2" were created. These sentences were rated as difficult to comprehend by two judges. An example incomprehensible sentence created in this way would be, "The breakfast was excellent because the needle on the Christmas tree fell." Care was taken to ensure that no two clauses from the same comprehensible sentence were merely placed in reverse order in a recombined sentence.

Two test lists, to be masked by white noise, were constructed. These lists were formed in such a way that half the sentences in each list were replicas of comprehensible acquisition sentences and half were replicas of incomprehensible acquisition sentences. (In addition, no clause was repeated within a test list.) The test lists were counterbalanced so that each sentence from both acquisitions appeared once in intact form and once in recombined form. Each test list was recorded on tape.

Procedure. The volume level of the test tapes was set at normal conversational level. The level of white noise used to mask the targets was varied systematically in a pilot study until naive subjects could identify approximately $45 \%$ of the targets correctly.

There were three experimental groups in the experiment. Subjects in the baseline group received the white-noise test without any prior acquisition experience. They were told the number of each sentence before it occurred (to serve as a ready signal). Following presentation of each masked sentence, the tapes were turned off and subjects were allowed $25 \mathrm{sec}$ to write their response. Partial responses were encouraged. Two examples preceded the actual test to allow subjects to become familiar with the procedure.

Subjects in the comprehensible acquisition condition were read the list of comprehensible sentences prior to the whitenoise test, and subjects in the incomprehensible acquisition condition were read the set of recombined incomprehensible sentences. As a means of ensuring that the subjects attended to the acquisition sentences, both acquisition groups were instructed to mark on a provided sheet whether they understood the mean ing of the sentence read to them. Three categories were used: "understood," "not sure," and "not understood." As a control for primacy and recency effects, an extra acquisition sentence was presented at the beginning of the list and two additional sentences were added at the end of the list. These sentences were not part of the subsequent test.

Following both comprehensible and incomprehensible acquisition conditions, subjects were told that they would be listening to a series of masked sentences. They were informed that some of the sentences would be copies of those they had just heard and others would be composed of jumbled clauses from the acquisition sentences. The procedure for presenting the test sentences was identical to that in the baseline condition Note that for the comprehensible acquisition condition, the comprehensible test sentences were copies of the acquisition sentences and the incomprehensible test sentences were novel recombinations of the clauses from the acquisition sentences. The reverse was true for the incomprehensible acquisition condition.

\section{Results and Discussion}

The results of the comprehension test administered during acquisition revealed that subjects in the comprehensible acquisition condition reported that they understood the meaning of $94 \%$ of the sentences. Subjects in the incomprehensible acquisition condition reported understanding the meaning of only $20 \%$ of the acquisition sentences.
Table 1

Mean Percentage Correct Identification with

Standard Deviations for the Experimental Conditions in Experiment 1

\begin{tabular}{|c|c|c|c|c|}
\hline \multirow{3}{*}{$\begin{array}{c}\text { Types of } \\
\text { Acquisition }\end{array}$} & \multicolumn{4}{|c|}{ Test Sentence } \\
\hline & \multicolumn{2}{|c|}{ Comprehensible } & \multicolumn{2}{|c|}{ Incomprehensibl } \\
\hline & Mean & SD & Mean & SD \\
\hline Comprehensible & 76.5 & 11.9 & 68.6 & 9.1 \\
\hline Incomprehensible & 65.2 & 8.4 & 70.2 & 7.5 \\
\hline Baseline & 45.8 & 9.8 & 45.2 & 11.7 \\
\hline
\end{tabular}

Note $-N=12$ in each condition.

Results of the white-noise test were scored by the number of clauses each subject reproduced correctly. Scoring was based on the accurate reproduction of the nouns and verbs in the clauses, ignoring reproduction of "because" or articles. The results (given by the percentage of the clauses correctly reproduced) are presented in Table 1.

The data were analyzed by a 3 (baseline/comprehensible acquisition/incomprehensible acquisition) by 2 (test tapes) by 2 (comprehensible test sentences/incomprehensible test sentences) mixed-design analysis of variance, with the first two factors being the betweensubjects factors. Analyses were conducted over both subjects and words, and min $F^{\prime}$ statistics (Clark, 1973) were computed. A highly significant main effect due to type of acquisition was obtained $\left[\min \mathrm{F}^{\prime}(2,67)=23.13\right.$, $p<.001]$. The interaction between type of acquisition and type of test sentence was not significant with the $\min \mathrm{F}^{\prime}$ analysis $\left[\min \mathrm{F}^{\prime}(2,120)=2.24\right]$, although this interaction was significant for both $F_{1}$ and $F_{2}\left[F_{1}(2,30)\right.$ $\left.=6.80, p<.005 ; F_{2}(2,96)=3.35, p<.05\right]$. No other main effects or interactions were significant. The nonsignificance of the min $F^{\prime}$ for the Acquisition by Test interaction questions the status of integrated and nonintegrated propositional structures as episodic units in perceptual recognition. If integrated propositional structures were episodic units, then the comprehensible acquisition/comprehensible test condition should show greater reproduction accuracy than the comprehensible acquisition/incomprehensible test condition. Although the difference between these conditions was in the hypothesized direction, it was quite small (7.9\%). Similarly, if nonintegrated propositional structures were viable episodic units, then the incomprehensible acquisition/incomprehensible test condition should have demonstrated enhanced performance relative to the incomprehensible acquisition/comprehensible test condition. Again, although the difference was in the predicted direction, it was even smaller (5.0\%). It might be argued that since the $\min \mathrm{F}^{\prime}$ statistic is highly conservative (cf. Forster \& Dickinson, 1976), the fact that both the $F_{1}$ and $F_{2}$ interactions were significant indicates that one or both of the above small differences may in fact be real. However, even given these more liberal statistics, there is little support for integrated or nonintegrated 
propositional units. Using Dunn's multiple-comparison procedure (Kirk, 1968), neither the $F_{1}$ nor the $F_{2}$ comparison for nonintegrated propositional units was significant $\left[d=5.00 ; F_{1}\right.$ critical value: $d(30)=5.86$; $F_{2}$ critical value:d $\left.(48)=12.86\right]$. For the comparisons testing for integrated propositional units, only the $F_{1}$ comparison was significant $\left[\mathrm{d}=7.92 ; \mathrm{F}_{1}\right.$ critical value: $\mathrm{d}(30)=5.86 ; \mathrm{F}_{2}$ critical value: $\left.\mathrm{d}(48)=12.86\right]$. Thus, the present results do not support either integrated or nonintegrated propositional structures as episodic memory units that mediate enhanced perceptual recognition.

Next consider the status of concepís and propositions as episodic memory units. By hypothesis, if concepts and/or propositions are units of episodic memory, then the conditions in which words and clauses are held invariant while clause combinations are varied should show enhanced perceptual recognition. Two comparisons examined this possibility. The comprehensible acquisition/incomprehensible test condition demonstrated greater reproduction accuracy than the baseline/ incomprehensible test condition $\left[\min \mathrm{F}^{\prime}(1,119)=19.90\right.$, $p<.001]$. Also, the incomprehensible acquisition/ comprehensible test condition showed greater reproduction accuracy than the baseline/comprehensible test condition $\left[\min \mathrm{F}^{\prime}(1,119)=13.68, \mathrm{p}<.001\right]$. These comparisons examine the efficacy of the concept and/or proposition units without contamination due to possible effects of units composed of either integrated or nonintegrated propositions. Note that while the results of these comparisons suggest that concepts and/or propositions are effective cognitive units, the design of this first experiment does not allow differentiation of the effects due to these two types of units. Experiment 2 is designed to separate the effects due to concepts from those due to propositions.

\section{EXPERIMENT 2}

In overview, this experiment also consists of an acquisition phase followed by a white-noise test. However, in the present study, all acquisition and test stimuli consist of incomprehensible two-clause sentences. The logic of the design is the same as in the first experiment. For all acquisition conditions, concepts are held invariant between acquisition and test by presenting test sentences that contain the same content words as appeared in acquisition sentences. However, in this study, propositional units either are held invariant (clause invariance condition) or are altered between acquisition and test (word recombination condition).

As in Experiment 1, all test conditions receiving acquisition will be compared with a baseline condition that receives the white-noise test without prior acquisition. The general hypothesis is identical to that of the first experiment; if a given unit is effective, then facilitated reproduction accuracy will occur when that unit is held invariant between acquisition and test. In the present experiment, two specific expectations follow from this hypothesis: (1) If concepts are units of episodic memory, then all conditions receiving acquisition will exhibit facilitated reproduction when compared with baseline performance, since content words are held invariant between acquisition and test. (2) If propositions are episodic units, then test sentences that hold clauses invariant between acquisition and test will be more accurately reproduced than test sentences that break the clause structures by recombining content words from acquisition sentences.

\section{Method}

Subjects. Twenty-four undergraduate students at Vanderbilt University participated in this experiment as part of an introductory psychology course requirement.

Materials. The materials used were the 20 difficult-tocomprehend sentences used by Auble et al. (1979) plus the 6 additional difficult-to-comprehend sentences used in Experiment 1 . An example of one of these sentences would be "The family asked for rooms because the light had bumed out." For this experiment, the words enabling comprehension of the sentences to be achieved were not inserted into the sentences. Then, as in the incomprehensible acquisition condition in Experiment 1 , the clauses making up these sentences were recombined across sentences to form 26 new incomprehensible sentences. For instance, "The ends were too large because the family asked for rooms," was one of these sentences. These 26 incomprehensible sentences formed the test list. Two acquisition lists were created. For each acquisition list, half of the sentences in the test list were broken up into content words and recombined to form new incomprehensible sentences (word recombination condition). The two clauses in each of these new sentences were, as in the old sentences, grammatically and semantically correct and were connected by "because." None of the newly created sentences contained more than one content word from any one of the original sentences. An example of one of these recombined sentences would be "The man rotated because the smell disappeared." The clauses for the remainder of the acquisition list remained intact between acquisition and test (clause invariance condition). The acquisition lists were counterbalanced so that the recombined words in one list were the original clauses in the other list and vice versa. Thus, over both acquisition lists, each test sentence appeared in both clause invariance and word recombination conditions.

Procedure. The volume levels of the target tape and whitenoise mask were set as described in Experiment 1 . Baseline subjects were given the same instructions, time intervals, and practice as baseline subjects in the first experiment.

Subjects in the acquisition condition were given the same comprehension rating task, instructions, and score sheet that were used in Experiment 1. Half of the acquisition subjects were read one acquisition list and half were read the other acquisition list prior to the white-noise test. Testing involved the same reproduction task, time intervals, and test list as the baseline condition.

\section{Results and Discussion}

The results of the comprehension rating indicated that subjects generally did not understand the acquisition sentences. Overall, subjects reported that they did not understand or were unsure of the meaning of $75.0 \%$ of the sentences in the first acquisition list and 74.5\% of the sentences in the second acquisition list.

Results for the white-noise task were scored by the number of content words correctly reproduced by each 
subject. It was found that the mean correct reproduction rate for baseline subjects was $36.12 \%$ ( $\mathrm{SD}=7.27$ ). For the acquisition group, the mean for the clause invariance condition appeared substantially higher than baseline (mean $=51.19 \%, \mathrm{SD}=7.42$ ), whereas the mean for the word recombination differed little from baseline (mean $=39.62 \%, \mathrm{SD}=8.98$ ). The results from the data analysis confirmed these initial impressions.

A 2 (baseline/acquisition) by 2 (counterbalanced acquisition lists) by 2 (clause invariance/word recombination) mixed-design analysis of variance was conducted, with the first two factors varied between subjects and the third factor varied within subjects. Since baseline subjects received no acquisition, half were randomly assigned to each level of the acquisition list factor. Similarly, the factor "clause invariance/word recombination" is meaningless in the baseline condition. For baseline, data for each level of this factor were assigned so that the same test sentences would appear in the same conditions as in the acquisition group. Significant main effects were obtained for the baseline/ acquisition factor $\left[\min F^{\prime}(1,58)=12.64, p<.005\right]$ and the clause invariance/word recombination factor $\left[\min F^{\prime}(1,90)=5.44, p<.025\right]$. In addition, the interaction between these two factors was significant $\left[\min F^{\prime}(1,56)=6.27, p<.025\right]$. No other main effects or interactions were significant.

Two comparisons were performed to examine the specific hypotheses of this study. If concepts are episodic units, then the acquisition group should be superior to baseline for the word recombination condition. This comparison was not significant $\left[\min \mathrm{F}^{\prime}(1,128)<1\right.$ ], indicating that invariance of concept units did not lead to enhanced perceptual recognition. To examine the replicability of the results of Experiment 1 on the effectiveness of propositional units in facilitating perceptual recognition, and also to verify the sensitivity of the present experimental conditions to such effects, the clause invariance acquisition condition was compared with baseline. Accuracy rates in this condition were found to be significantly greater than for baseline $\left[\min \mathrm{F}^{\prime}(1,128)=23.07, \mathrm{p}<.001\right]$, indicating a facilitation effect due to clausal invariance. Thus it would appear that the results provide evidence supporting propositions as functional cognitive units of episodic memory. The results obtained under the present conditions do not indicate the occurrence of any effective facilitation due to concept units.

\section{GENERAL DISCUSSION}

The present studies were designed to examine the cognitive units underlying episodic memory. Four possible units were examined: concepts, propositions, integrated propositions, and nonintegrated propositions. The results of the two experiments indicate that only invariance of propositions leads to substantial facilitation effects in perceptual recognition. This evidence corroborates the general conclusions of other researchers (e.g., Anderson, 1974; Graesser, 1978; Ratcliff \& McKoon, 1978) concerning the importance of propositional units for a variety of cognitive tasks. The results of the present studies reveal little evidence for concepts as units of episodic memory. This finding may seem somewhat surprising, since, a priori, it would seem obvious that concepts must be cognitive units in some sense and, as such, must under some conditions be mediators of episodic memory. In fact, pilot studies we have conducted indicate that presenting subjects an acquisition list of single words does effectively enhance the reproduction of those words in subsequent whitenoise tests. Also, Jacoby and Dallas (in press) found enhanced perceptual recognition for previously presented single words. However, in both of these cases, the experimental tasks were oriented toward single words, not words in more elaborate linguistic structures. The present results indicate that when words are presented within such elaborated linguistic structures (i.e., clauses), the words become contextualized. That is, the concepts become intrinsic components of propositional structures and may not have the status of units independent of these structures. A number of studies have demonstrated decremental effects of contextual change on episodic memory performance (e.g., Barclay, Bransford, Franks, McCarrell, \& Nitsch, 1974; Tulving, 1979). The present finding seems to be a related effect.

With respect to such contextual effects, it is interesting to note that propositions, unlike concepts, do not seem to lose their status as independent units when they are integrated into higher order structures. Propositional invariance affects reproduction accuracy even when the sentential context is altered by recombining clauses. To some extent, this maintenance of propositions as independent units may be due to the particular sentential structures used in the present experiments (i.e., two conjoined clauses). Further work can examine the generality of this independence by exploring different sentence structures. However, pending this future work, the present findings do provide tentative support for the conclusion that propositions are primary episodic memory units for linguistic structures more complex than single words. Similarly, although the present work provides little evidence that integrated propositional structures are effective episodic units, an interesting area for future research will be to examine whether this finding generalizes to other types of sentence structures.

Although the present studies were oriented toward assessing the episodic units mediating perceptual recognition, an additional question concerns the processes by which such mediation takes place. Two conceptualizations will be considered. One is based on an extension of the findings of Jacoby and Dallas (in press); the other is related to the work on cognitive units by Ratcliff and 
McKoon (1978). Jacoby and Dallas suggest that enhanced perceptual recognition of words is based on increased perceptual fluency. This fluency is attributed to prior episodic exposure to the physical characteristics of the words. Jacoby and Dallas' proposal can be extended to account for the present findings. It might be that subjects maintain an episodic encoding of the physical characteristics of whole clauses as single units. Such prior encodings might then increase the perceptual fluency in processing these clauses when they remain invariant at time of test.

A second possible process underlying the obtained enhanced perceptual recognition involves an extension of the findings of Ratcliff and McKoon (1978). Using a priming paradigm, these investigators demonstrated that presentation of one word (from a previously experienced proposition) primed the recognition reaction time for a second word from within that same proposition. The present results may be due to a similar effect. Upon presentation of a masked test sentence, subjects will correctly perceive certain words in the sentence even without the benefits of prior exposure. It may be that these correctly perceived words can then prime the perceptual recognition of other words from the same acquisition proposition. This priming would, of course, be useful only when propositions are held invariant between acquisition and test.

Since the present experiments were designed to examine structural units rather than processes, differentiation between these alternatives remains a question to be investigated in further studies. However, the data can provide evidence concerning a third possible explanation of the present findings. An anonymous reviewer of an earlier draft of this paper suggested that the present results might not be due to enhanced perceptual recognition at all but, rather, might be due to a cued recall guessing strategy. That is, a subject may remember some of the proposition from acquisition. Then during testing, a word from such a proposition might be correctly perceived, but the subject misses the remaining words in the test proposition. In such a case, the subject may guess the missed words by inserting the remainder of the remembered acquisition proposition that contained the correctly perceived word. This strategy would lead to greater accuracy in reproduction of invariant propositions without resulting in any enhancement of perceptual recognition processes per se. However, this guessing strategy would also lead to numerous intrusions from acquisition propositions in the word recombination condition. That is, when a single word from a word recombination test sentence is correctly perceived, a subject should incorrectly insert the remainder of the remembered acquisition proposition for the missed words. In fact, the occurrence of such intrusions is extremely low. Only three of these intrusions occurred. All three intrusions involved incorrectly reproducing "the party was small" (the acquisition proposition) instead of "the party was stalled" (the test proposition). It seems unlikely that even these three intrusions were due to the above guessing strategy. Due to the close phonological similarity between "small" and "stalled," such an incorrect substitution would be expected to occur given either of the earlier discussed possible processes underlying enhanced perceptual recognition.

Thus, the evidence suggests that the present results are indeed due to enhanced perceptual recognition. Furthermore, the results indicate that propositions are the primary episodic units that mediate perceptual recognition for linguistic structures more complex than single words.

\section{REFERENCE NOTE}

1. Anderson, J. R. Concepts, propositions, and schemata: What are the cognitive units? Colloquium presented at Vanderbilt University, Nashville, Tennessee, November 1979.

\section{REFERENCES}

ANDERson, J. R. Verbatim and propositional representation of sentences in immediate and long term memory. Journal of Verbal Learning and Verbal Behavior, 1974, 13, 149-162.

Auble, P. M., Franks, J. J., \& Soraci, S. A. Effort toward comprehension: Elaboration or "ahal"? Memory \& Cognition, $1979,7,426-434$.

Barclay, J. R., Brangford, J. D., Franks, J. J., McCarrell, N. S., \& Nitsch, K. Comprehension and semantic flexibility. Journal of Verbal Learning and Verbal Behavior, 1974, 13, 471-481.

Clark, H. H. The language-as-fixed-effect fallacy: A critique of language statistics in psychological research. Journal of Verbal Learning and Verbal Behavior, 1973, 12, 335-359.

Graesser, A. C. Tests of a holistic chunking model of sentence memory through analyses of noun intrusions. Memory \& Cognition, 1978, 6, 527-536.

Forster, K. I., \& Dickinson, R. G. More on the language-asfined-effect fallacy: Monte Carlo estimates of error rates for $F_{1}$, $F_{2}, F^{\prime}$, and min $F^{\prime}$. Journal of Verbal Learning and Verbal Behavior, 1976, 15, 135-142.

Hannigan, M. L., Shelton, T. S., Franks, J. J., \& Brangrord, J. D. The effects of episodic and semantic memory on the identification of sentences masked by white noise. Memory \& Cognition, 1980, 8, 278-284.

Jacogy, L. L., \& Dallas, M. On the relationship between autobiographical memory and perceptual learning. Journal of Experimental Psychology: General, in press.

KIRK, R. E. Experimental design: Procedures for the behavioral sciences. Belmont, Calif: Brooks/Cole, 1968.

Ratcliff, R., \& McKoon, G. Priming in item recognition: Evidence for the propositional structure of sentences. Journal of Verbal Learning and Verbal Behavior, 1978, 17, 403-417.

Tulving, E. Episodic and semantic memory. In E. Tulving \& W. Donaldson (Eds.), Organization of memory. New York: Academic Press, 1972.

Tulving, E. Relation between encoding specificity and levels of processing. In L. S. Cermak \& F. I. M. Craik (Eds.), Levels of processing and human memory. Hillsdale, N.J: Erlbaum, 1979.

\section{NOTE}

1. Technically, it could be argued that some of the clauses used, like "the parachute cloth ripped" or "the bagpipe split," are not represented by simple propositions. However, most of the clauses used clearly are representable by simple propositions. More important for present purposes, the level of structure corresponding to the clauses is clearly distinct from the other possible levels of structure investigated, and "proposition" appears to be the generally accepted term that most clearly corresponds to the clauses. 


\section{Appendix}

Comprehensible Sentences Used in Experiment 1

1. The child was hungry because the fishing pole splintered.

2. The table did not move during the seance because the wire snapped.

3. The haystack was important because the parachute cloth ripped.

4. The igloo was small because the sun came out.

5. The crowd was disappointed because the hangman's knot slipped.

6. The family asked for rooms because the no vacancy light had burned out.

7. The breakfast was excellent because the spiderweb was sticky.

8. The meat was burned because the clock stopped.

9. The man's back ached because the barbells were too large.

10.The girl spilled her popcorn because the lock on the lion cage broke.

11.The boy was pleased because the rabbit disappeared during the magic act.

12.The street was full of holes because the cement mixer stopped turning.
13. The person did not rise because the elevator door was jammed.

14. The food was intact because the dentures fell apart.

15. The tree grew because the peach pit opened.

16.The party was stalled because the corkscrew straightened.

17. The smell began because the garbage strike signs went up.

18. The lady rose because the balloon expanded.

19. The clothes were ruined because the wet paint sign vanished.

20.The marionettes embraced because the strings tangled.

21. The notes were sour because the bagpipe split.

22 . The audience cheered because the juggler rotated five balls.

23. The needle on the Christmas tree fell because the bucket was not filled.

24. The person was unhappy because her pierced ears closed.

25. The woman could not see because the windshield wiper blade was bent.

26. The dinner was uneaten because the chopsticks were warped.

(Received for publication February 26, 1981; revision accepted July 14, 1981.) 